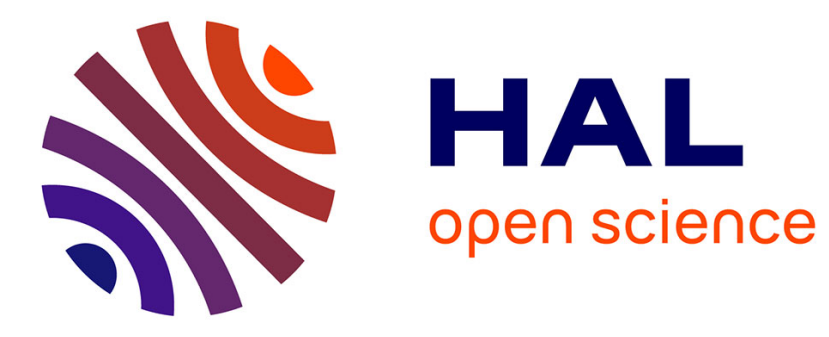

\title{
Perspectives asiatiques sur les Smart Cities
}

\author{
Carine Henriot, Nicolas Douay, Benoît Granier, Raphaël Languillon-Aussel, \\ Nicolas Leprêtre
}

\section{To cite this version:}

Carine Henriot, Nicolas Douay, Benoît Granier, Raphä̈l Languillon-Aussel, Nicolas Leprêtre. Perspectives asiatiques sur les Smart Cities. Flux - Cahiers scientifiques internationaux Réseaux et territoires, 2018, 114, pp.1-8. 10.3917/flux1.114.0001 . halshs-01991584

\section{HAL Id: halshs-01991584 https://shs.hal.science/halshs-01991584}

Submitted on 18 Jul 2019

HAL is a multi-disciplinary open access archive for the deposit and dissemination of scientific research documents, whether they are published or not. The documents may come from teaching and research institutions in France or abroad, or from public or private research centers.
L'archive ouverte pluridisciplinaire HAL, est destinée au dépôt et à la diffusion de documents scientifiques de niveau recherche, publiés ou non, émanant des établissements d'enseignement et de recherche français ou étrangers, des laboratoires publics ou privés. 


\section{PERSPECTIVES ASIATIQUES SUR LES SMART CITIES}

Carine Henriot, Nicolas Douay, Benoit Granier, Raphaël Languillon-Aussel et Nicolas Leprêtre

Université Paris-Est Marne la Vallée | « Flux »

2018/4 N 114 | pages 1 à 8

ISSN 1154-2721

Article disponible en ligne à l'adresse :

https://www.cairn.info/revue-flux-2018-4-page-1.htm

Distribution électronique Cairn.info pour Université Paris-Est Marne la Vallée.

(C) Université Paris-Est Marne la Vallée. Tous droits réservés pour tous pays.

La reproduction ou représentation de cet article, notamment par photocopie, n'est autorisée que dans les limites des conditions générales d'utilisation du site ou, le cas échéant, des conditions générales de la licence souscrite par votre établissement. Toute autre reproduction ou représentation, en tout ou partie, sous quelque forme et de quelque manière que ce soit, est interdite sauf accord préalable et écrit de l'éditeur, en dehors des cas prévus par la législation en vigueur en France. Il est précisé que son stockage dans une base de données est également interdit. 


\title{
Perspectives asiatiques sur les Smart Cities
}

\author{
Carine Henriot \\ Nicolas Douay \\ Benoit Granier \\ Raphaël Languillon-Aussel \\ Nicolas Leprêtre
}

$\mathrm{D}$ epuis le tournant des années 2010, on observe une multiplication des discours et des expérimentations sur les "smart cities" (ou villes intelligentes) dans la plupart des pays du monde. En effet, dans le sillage des stratégies d'entreprises comme IBM, Huawei, Tencent, Hitachi ou Toshiba, les grandes métropoles internationales comme de nombreuses villes régionales affichent désormais leur prétention à devenir (plus) «smart». Les définitions comme la terminologie recouvrant l'ensemble des expérimentations de smart cities sont nombreuses. La ville intelligente est donc plurielle avec toutefois quelques constances comme l'ont souligné Picon (2013), Townsend (2014), Douay (2018a) ou encore Greenfield (2013). Ces auteurs notent une opposition entre les approches top-down souvent inspirées par une croyance dans la puissance des systèmes techniques et des approches bottom-up d'orientation plus collaborative. À l'échelle globale, la diffusion de la ville intelligente, comme nouveau « modèle » de développement urbain, entraîne un «basculement de la polarité du développement vers le sud-est » (Rochet, 2014) - soit vers I'Asie. C'est de ce basculement que nous souhaitons rendre compte: en ouvrant plusieurs perspectives asiatiques sur les smart cities à partir de l'observation de différentes configurations territoriales, aux échelles nationales et urbaines.

L'Asie est I'une des régions du monde comptant le plus de projets de smart cities, près du tiers selon les estimations de Coll (2014). En Chine continentale, par exemple, ce sont plus de 300 initiatives qui ont été mises en place depuis 2011, plus de 500 d'ici 2020. En Inde, le programme «Smart Cities Mission » publie dès 2015 un appel à projet pour sélectionner et promouvoir 100 villes intelligentes indiennes. Toujours en 2015, ce sont plus de 200 expérimentations qui sont dénombrées au Japon (DeWit, 2014). Cette impressionnante statistique conduit à interroger les caractéristiques " asiatiques » favorisant l'émergence et la multiplication de ces initiatives, par-delà les inégalités régionales et nationales de développement, mais également par-delà les différences de régimes politiques. Quelles conditions asiatiques président à la programmation de ces villes intelligentes? Quelles trajectoires les smart cities asiatiques dessinent-elles? Présentent-elles des caractéristiques communes, comme par exemple la prévalence pour le hardware ou au contraire pour le software? Quelle place est attribuée à la technologie, aux usages qui en sont faits, à la participation citoyenne?

Ce dossier thématique se propose d'approcher les politiques asiatiques de villes intelligentes par leur action publique et les stratégies d'acteurs qui leur sont adossées, et au-delà, interroge la place de cette partie du monde dans la structuration d'un éventuel modèle asiatique de la ville intelligente.

\section{LES SMART CITIES AU PRISME DE L'ASIE}

Le présent dossier se réfère à l'Asie dans un sens large et dépolitisé, comme cette partie du monde à l'Est de l'Europe. Cette Asie se signale par une urbanisation rapide et récente, une production urbaine renouvelée rendue possible par une importante financiarisation de la ville. L'implication très répandue du secteur privé dans l'aménagement urbain de plusieurs pays, comme le Japon, la Corée, la Chine, mais aussi Singapour ou encore Taiwan et Hong Kong a conduit, plus rapidement peut-être qu'en Europe, à l'arrivée dans le champ de la fabrique urbaine des acteurs du numérique, de l'informatique et des 
nouvelles technologies de l'information et de la communication. L'Asie, ses territoires et ses populations, cumulent en effet un engouement pour le numérique, rendant la région particulièrement réceptive aux innovations technologiques, urbaines et numériques. Rochet (2014) souligne en outre combien «les enjeux du développement de villes intelligentes sont ceux de I'industrialisation des pays émergents (...). Ces pays n'ont pas la technologie mais ont une vision stratégique et politique du développement urbain » (p. 14).

La mobilisation du mot d'ordre smart dans I'aménagement urbain asiatique se trouve ainsi à la confluence de plusieurs phénomènes : la diffusion d'un marketing et de solutions technologiques par plusieurs grandes firmes globalisées comme IBM et Toshiba (Languillon-Aussel, Leprêtre, Granier, 2016), la réutilisation de ce vocable et de ces solutions par des États et collectivités territoriales souhaitant innover et s'approprier des «bonnes pratiques» et, en toile de fond, l'impérieuse nécessité d'une transition énergétique et écologique pour laquelle les modalités d'action concrète sont encore à définir. Cette tendance est confortée par une production urbaine dynamique dans la région, un engouement pour le numérique et une action publique agile. Dix ans après la popularisation de la stratégie Smarter Cities lancée en 2008 par IBM, il est délicat de formuler un premier bilan d'une pluralité d'initiatives qui oscillent entre expérimentation, nécessairement ponctuelle, et plan d'action à plus long terme, entre marketing territorial ambitieux et continuité de plans de rénovation thermique auxquels est greffée une " couche » de numérique. Ainsi, si la promesse de la smart city est porteuse de nouveauté dans l'aménagement urbain par les services qu'elle propose, ce dossier met en lumière la continuité du processus de fabrique de la ville en Asie, tout en ouvrant des pistes de réflexion pour essayer de qualifier l'engouement récent pour le champ lexical du smart appliqué aux espaces urbains asiatiques des années 2010.

\section{Pour des Perspectives diversifiées SUR LA MISE EN INTELLIGENCE DES VILLES ASIATIQUES}

Les aménagements de smart city se sont rapidement multipliés en Asie dans des aires géographiques variées, portées par des contextes politiques et des niveaux de développement tout aussi contrastés. Les initiatives smart se multiplient tout autant dans les pays les plus industrialisés, comme le Japon ou la Corée, les cités étatiques, elles-mêmes très avancées dans leur développement industriel, technologique et, en partie, démocratique, comme Singapour ou Hong Kong, les pays émergents, comme I'Inde ou la Chine, ainsi que les pays actuellement en développement, comme le Vietnam. Le présent dossier propose une mise en perspectives asiatique sur les Smart Cities à partir de trois initiatives japonaises, deux chinoises, I'une continentale l'autre à Hong Kong, et une initiative indienne.

\section{Les références au Smart : un vocable incontournable qui circule rapidement en Asie}

L'arrivée du smart en Asie se signale tout d'abord par l'introduction et la stabilisation de l'appellation par les entreprises internationales, puis la traduction dans plusieurs langues asiatiques, de cette mise en intelligence, et de leur définition par des acteurs gouvernementaux.

En Inde, où l'anglais reste la deuxième langue officielle, le vocabulaire lié au smart est directement introduit et utilisé en anglais. Il est officiellement usité par le programme « 100 smart cities mission », lancé en 2015.

En Chine continentale, par exemple, IBM lance en 2008 un plan intitulé "Breakthrough of smart city in China », à I'heure où la notion de développement durable percole dans le vocabulaire officiel et les projets d'aménagement urbain chinois (Douay, Henriot, 2016). Fin 2011, le ministère chinois de la Technologie et la Commission européenne établissent un référentiel sino-européen d'expertise sur la ville intelligente qu'ils choisissent de nommer «Green Smart City »). Dès 2012, un appel à projet, lancé par le ministère du Logement et du Développement urbain et rural, vise à identifier les modèles pilotes de « villes intelligentes" (zhihuì chéngshì).

Toutefois, le terme smart city ne s'est pas imposé partout de la même manière, et ne désigne pas nécessairement le même objet urbain, ni la même configuration d'acteurs. Au Japon, par exemple, c'est avant tout l'appellation de smart community (sumāto komyuniti) qui prévaut pour le gouvernement japonais, et non smart city (sumāto shiti). En effet, en 2010, le terme communauté intelligente désigne le premier programme de démonstrateurs financés par le ministère de l'Économie, du Commerce et de l'Industrie, et s'est depuis imposé. Cependant, ces mêmes démonstrateurs gouvernementaux japonais ne différencient pas toujours cette appellation de l'emploi d'autres syntagmes, comme smart city ou eco city. Les projets financés par le secteur privé, quant à eux, reprennent aussi cette diversité d'appellations. L'usage du vocabulaire anglais, bien qu'assez 
courant dans la langue japonaise, s'explique par la transposition de concepts de smart grid et de smart city produits par des firmes étatsuniennes comme IBM, en concurrence directe avec les conglomérats japonais.

Au-delà du vocabulaire, il est systématiquement fait référence à des démonstrateurs techniques.

\section{Des smart cities résolument technicistes}

Une approche par l'ingénierie urbaine, majoritaire en Asie, conçoit en outre les smart cities comme des villes structurées par la gestion instantanée du big data, ou métadonnées, issues de la technologisation de l'urbain et de la mise en œuvre de solutions numériques dans les espaces urbains et les réseaux. Les villes deviendraient smart lorsqu'elles permettraient le recours au traitement informatique simultané de l'information afin de gérer et d'anticiper la dynamique des espaces, des réseaux et des populations. Une approche entre modélisation et ingénierie informatique détermine la smart city comme reposant sur I'usage massif de capteurs, de super-calculateurs et de l'Internet, afin de connaitre et de gérer la ville sur le très court terme (Batty, 2013).

Le smart investit ainsi de façon privilégiée les réseaux, à travers l'optimisation de la gestion des différents services urbains, participant de cette approche néo-positiviste : électricité, gaz, eau et assainissement, déchets, réseaux de chaleur urbains, mais aussi transports (Dupuy, 2014). Ainsi par exemple, la production, la distribution et la consommation de l'énergie sont alors optimisées par l'usage de "réseaux électriques intelligents » dits smart grid qui, grâce à des technologies informatiques, ajustent les flux d'électricité aux besoins (Sechilariu, Wang, Locment, 2013). Le développement par les acteurs des NTIC de systèmes de gestion des flux d'électricité, de chaleur et d'eau à l'échelle de la ville, du quartier voire du bâtiment, pose la question d'une rupture avec le modèle réticulaire des macro-systèmes techniques (Coutard, Rutherford, 2009) et de la généralisation de systèmes de production décentralisée (Erlinghagen, Markard, 2012). L'usage dans les transports $d^{\prime}$ 'une signalisation numérique en temps réel relayant les informations fournies par des capteurs embarqués (boucles de rétroaction), voire le couplage transports intelligents et énergies renouvelables, rendent alors la ville à la fois plus numérique et plus durable, donc "smarter ", tout en la plaçant au service du citoyen (Albino, Berardi, Daneglico, 2015). Parmi les pays étudiés, cette approche est la plus prégnante au Japon, où les enjeux de planification urbaine comptent moins que l'expérimentation de systèmes sociotechniques visant à optimiser en temps réel la production et la consommation d'énergie (voir Nicolas Leprêtre, 2018, ce dossier). À cet égard, le CEMS (Community Energy Management System), déployé par les entreprises privées avec le soutien du gouvernement, constitue le parangon d'une gestion centralisée et informatisée de flux complexes (production, consommation, stockage, échange d'énergie).

Néanmoins, au-delà du débat relatif à la compatibilité de la recherche d'un accroissement continu de la croissance économique avec la réduction drastique des émissions de gaz à effet de serre, la mise en avant constante de la hausse de la qualité de vie dans les «smart » cities pose question (Caragliu, Del Bo, Nijkamp, 2009; Giffinger et alii, 2007). Plusieurs recherches fondées sur les théories de la pratique sociale (social practice theories) ont récemment mis en avant la difficulté voire l'impossibilité de prédire l'impact de l'introduction de nouvelles technologies dans l'espace domestique (Gram-Hanssen, 2011), ces dernières risquant d'augmenter voire de créer des normes de confort toujours plus intensives en énergie (Shove, 2010). Dans cette optique, Strengers (2013) qualifie l'ambition de parvenir à un développement durable grâce aux «smart energy technologies » de « Smart Utopia ».

\section{Une fabrique urbaine par l'expérimentation}

Le recours à "l'expérimentation» n'est pas une nouveauté dans la conduite des politiques d'innovation. Cette pratique s'inscrit le plus souvent dans des " niches " protégées et limitées dans le temps afin de s'assurer du fonctionnement technologique et de la viabilité $d^{\prime}$ un modèle économique face à des investissements lourds (Rip, Kemp, 1998; Erlinghagen, Markard, 2012). Il semble toutefois qu'il s'opère depuis les années 2000 un recours de plus en plus récurrent à cet instrument d'action publique dans la conduite des politiques environnementales et énergétiques, comme l'ont pointé Bulkeley et Castán Broto (2013).

Ce "gouvernement par l'expérimentation» comme le désignent les auteurs accorde une place importante à l'interaction des systèmes sociotechniques avec la population pour pointer les éventuelles failles ou résistances, tout en identifiant les « bonnes pratiques » à répliquer. Le territoire urbain serait ainsi érigé comme "vitrine» du volontarisme politique. La multiplication de démonstrateurs de smart cities en Asie tend à 
conforter cette hypothèse et invite à réfléchir sur le rôle conféré aux acteurs publics et privés, mais aussi aux utilisateurs, dans la production et I'utilisation d'infrastructures « intelligentes » ou connectées. L'expérimentation pose également la question de la généralisation des systèmes à l'échelle de la ville ou d'un territoire plus large, ou au contraire d'une fragmentation qui tendrait à une hybridation entre macro-système technique et micro-réseaux (Coutard, Rutherford, 2009).

Dans l'étude des projets urbains, Hernandez (2015) a explicité les conditions d'élaboration et de formalisation des contenus. À rebours des principes de linéarité et de hiérarchie entre objectifs et moyens, les projets urbains consistent souvent par assemblages de projets opérationnels décidés antérieurement, parfois en cours de réalisation. L'exercice de planification s'appréhende alors comme un "événement» au cours duquel les interactions entre différents projets et scènes d'action, initialement hétérogènes et autonomes avec différents objectifs et moyens, structurent l'émergence d'une signification d'ensemble : un projet.

En transposant cela aux contextes d'émergence des smart cities, nous pouvons faire l'hypothèse que cet assemblage de projets et d'actions venant de la sphère publique mais aussi des acteurs privés, répondant à différents objectifs sectoriels de l'action publique, participe finalement à l'avènement d'un nouveau référentiel ou plutôt label pour la pratique contemporaine de l'aménagement de l'espace qui dessine autant de configurations locales (Douay, 2018a).

Ces expérimentations de villes intelligentes en Asie, mais aussi au-delà, correspondent à l'approche critique, défendue par exemple par Greenfield (2013), selon lequel les projets de smart cities participent à une logique capitaliste qui perpétue la croissance économique en fournissant de nouveaux marchés aux plus grands groupes privés, comme IBM, Cisco, Veolia, Dassault, General Electric, Siemens, Phillips pour les groupes occidentaux, mais aussi des groupes asiatiques comme Samsung, LG, Toshiba, Panasonic, Tencent, Huawei ou Alibaba. À cet égard, le cas de Hong Kong est intéressant car la production urbaine est dominée par de grands conglomérats qui dominent le secteur immobilier mais n'ont pas réellement élargi leurs activités au domaine des innovations technologiques. Il s'agit là d'une des explications de la faiblesse de l'intensité technologique des projets smart dans l'ancienne colonie britannique (voir Nicolas Douay, 2018b, ce dossier).

\section{Des politiques publiques volontaristes, voire développementalistes}

La diversité des projets de villes intelligentes s'inscrit aussi dans une approche par les politiques. En effet, les acteurs publics aux échelles locales comme nationales restent au centre des configurations territoriales, notamment pour impulser les dynamiques qui sont ensuite plutôt mises en œuvre par le partenariat avec les acteurs privés. Cependant, l'intensité technologique des changements peut être très variable et renvoyer à des motivations politiques qui peuvent dépasser l'agenda digital.

À Hong Kong, le thème de la ville intelligente est devenu un vocabulaire dominant dans les discours portés par les responsables politiques et techniques de I'urbanisme. À cet égard, la reconversion de l'ancien aéroport donne lieu à la mise en œuvre d'un projet, Kowloon East, qui s'inscrit dans tous les mots d'ordre de la fabrication urbaine contemporaine sans forcément renvoyer à une rupture technologique. Dans ce sens, le numérique correspond plutôt à un renouvellement de la narration, c'est-à-dire des formes de mise en récit des pratiques de l'urbanisme et ouvre la possibilité de nouvelles expérimentations au-delà même des seules innovations numériques (voir Nicolas Douay, 2018b, ce dossier). Toutefois, ces évolutions n'impactent pas radicalement les contenus des projets et encore moins les rapports de pouvoir dans l'aménagement, voire conduisent au renforcement de la dépolitisation des enjeux urbains.

Au Japon, les formes que prennent la «smart city» ou "smart community» selon les appellations tiennent pour beaucoup à l'implication conjointe de municipalités volontaristes, de conglomérats japonais globalisés, mais aussi et surtout de l'État japonais. Le succès des expérimentations privées s'explique en partie par les jeux de bascules du pouvoir (décisionnel, organisationnel, financier, politique...) au sein des institutions, des organisations, des acteurs et des coalitions de croissance impliquées dans l'aménagement et la fabrique de l'urbain au Japon (voir Raphaël Languillon-Aussel, 2018, ce dossier). À travers un agenda orienté vers la compétitivité économique des firmes nationales, le gouvernement japonais impulse dès 2010 un " modèle national » de "smart community » qui comporte un ensemble de technologies liées à la consommation énergétique, comme des réseaux électriques intelligents ou des systèmes d'électromobilité (voir Nicolas Leprêtre, 2018, ce dossier). L'opportunité de ces technologies fut alors moins de gérer un réseau électrique qui, jusqu'à l'accident de Fukushima, demeurait performant, que d'inciter les 
collectivités territoriales à innover et promouvoir les bonnes pratiques, de créer des parts de marché pour les firmes nationales, et de tester de nouvelles politiques de changement des comportements (voir Benoit Granier, 2018, ce dossier).

En Chine continentale, comme ailleurs, les initiatives de smart city relèvent des politiques publiques mises en place par des appels à candidatures et bénéficient d'un fort encadrement par différentes entités étatiques, mises en concurrence, tandis que les collectivités territoriales soutiennent, orchestrent, et financent via des entreprises municipales publiques et privées, ces politiques liées à l'innovation. Le vocable de smart city est d'abord utilisé par des entreprises privées, avant qu'une réflexion autour de la smart city ne soit encadrée par l'équivalent chinois du Commissariat au Plan, la Commission pour le Développement national et la Réforme. Plusieurs programmes étatiques permettent d'explorer les différentes facettes de la smart city. Puis, selon une planification programmatique propre à la Chine, un appel à projet, destiné à labelliser les initiatives "modèles", est alors publié par le ministère du Logement et du Développement urbain et rural. Cette diffusion est alors censée bénéficier à l'ensemble du territoire chinois, là où la ville intelligente expérimentée jusqu'alors restait implantée dans les mégapoles littorales. En Chine, la smart city est introduite par IBM, avant que ce grand groupe international du hardware ne s'efface devant la montée en puissance de groupes chinois continentaux, d'abord relevant du domaine du hardware, comme Tencent, puis relevant du software et étrangers aux solutions urbaines, comme Alibaba (voir Carine Henriot, 2018, ce dossier).

En Inde, le projet 100 Smart Cities Mission est lancé en 2015. L'analyse du processus de sélection des villes, d'élaboration des plans d'aménagement et du détail des programmes d'investissements (voir Sama Khan, Persis Taraporevala et Marie-Hélène Zérah, 2018, ce dossier) rend compte du basculement d'une politique sectorielle vers une politique territorialisée. Derrière la mission, une large panoplie d'interventions est ainsi déployée : hybridation entre choix techniques, modalités de financement et stratégies d'élargissement des compétences ou d'externalisation. La contribution souligne également l'effet accélérateur des potentialités de partenariats avec le secteur privé dans la phase d'opérationnalisation des projets, ainsi que la systématisation du recours au conseil dans I'aide à la décision. Les injonctions à la participation citoyenne sont quant à elles bien moins suivies. Selon les auteurs, la diversité des trajectoires en formation de la ville intelligente est concomitante à la dépolitisation des politiques urbaines indiennes.

\section{Des habitants discrets pour des territoires voulus plus intelligents}

Si une abondante littérature porte sur les dispositifs techniques (numériques, algorithmiques, etc.) de la smart city, le sujet du rôle des habitants dans cette forme urbaine émergente n'en est pas moins fréquemment au centre des discours des acteurs. Les municipalités, en particulier, mettent en avant les nouvelles opportunités de participation citoyenne rendues possibles par le numérique et les technologies connectées. Les travaux de conceptualisation s'accordent également pour faire de la participation des habitants un élément constitutif de toute smart city (Hollands, 2008 ; Meijer, Rodriguez Bolivar, 2016; Mellouli, Luna-Reyes, Zhang, 2014). Dans une autre perspective, certaines études mettent en avant la dimension sécuritaire et disciplinaire de smart cities dont l'objectif serait davantage de changer les comportements des habitants, sans les faire participer à la gouvernance urbaine (Granier, Kudo, 2016 ; Vanolo, 2014). Plusieurs contributions du présent dossier s'attachent à analyser la forme concrète du rôle assigné aux habitants, et effectivement endossé par eux, dans les projets de smart cities. L'article de Benoit Granier analyse comment les smart cities japonaises, par contraste avec les discours sur la participation citoyenne, sont le support de dispositifs de changement des comportements ayant pour objectif de réduire et déplacer la consommation d'énergie des habitants. Les contributions de Nicolas Douay et de Sama Khan, Persis Taraporevala et MarieHélène Zérah montrent quant à elles que les projets de smart city hongkongais et indiens ne se traduisent pas non plus par davantage de participation des citoyens à la prise de décisions, et soulèvent la question de la dépolitisation des enjeux urbains, tandis que I'article de Raphaël Languillon-Aussel conclut sur le risque d'un renforcement des logiques de domination entre acteurs, mais aussi entre le secteur privé et le secteur public ou les habitants via la maîtrise des systèmes d'information urbains. Ces premiers résultats posent la question d'une particularité des smart cities asiatiques, ou d'un décalage plus général entre la vision idéale et les déclinaisons concrètes de la smart city.

\section{Vers un modèle asiatique de Smart City ?}

Globalement, les smart cities asiatiques étudiées dans ce dossier laissent entrevoir plusieurs éléments de convergence. Tout d'abord, un certain solutionnisme technologique combiné à 
l'aménagement d'infrastructures et la rationalité des plans inscrivent la ville intelligente asiatique dans la continuité d'un " urbanisme algorithmique » (Douay, 2018a). La prégnance de l'innovation et la rhétorique de la durabilité constituent des stratégies de distinction des territoires, des entreprises, mais aussi des États porteurs d'initiatives smart. Les liens existants entre stratégie étatique et conglomérats nationaux ou municipaux, dans le cas de Hong Kong et de Singapour, rappellent également les démarches développementalistes de la seconde moitié du XX ${ }^{\mathrm{e}}$ siècle. Par ailleurs, ces éléments de convergence induisent une caractéristique commune: la présence d'un État fort à l'initiative d'expérimentations smart et la place finalement discrète accordée aux habitants de ces quartiers intelligents.

Ces éléments de convergence font-ils pour autant consensus en Asie ? Pouvons-nous affirmer qu'un modèle asiatique de ville intelligente se structure? Si « le modèle consiste en un plan standard élaboré a priori pour promouvoir un ensemble de conditions sociales et matérielles tenues pour universellement valables : c'est un objet définitif et, par définition reproductible, quel que soit le contexte physique et social de son application »(Choay, 2015, p. 472), alors force est de constater que la smart city, par la multiplicité des visages qu'elle revêt, échappe en tant que tel à la généralisation, à la réplicabilité, au modèle. Pour autant, le modèle se situe également dans le registre des représentations et non dans le monde du réel ; il relève puissamment du langage et de la culture. Chaque État définit son idéal smart, son utopie intelligente, en teste les déclinaisons concrètes, technologique, systémique, en termes de services, puis promeut, par une programmation, d'échelle nationale ou municipale, donc re-diffuse un "modèle national » asiatique de ville intelligente.

Par ailleurs, la prépondérance des gouvernements centraux dans la formalisation de politiques industrielles "smart», la restructuration des gouvernances urbaines, la modification des coalitions de croissance locale, interrogent la dimension politique des smart cities et les enjeux de pouvoir derrière leur aménagement et leur diffusion rapide en Asie. À la fois pris dans une logique top-down des politiques publiques et bottom-up des coalitions d'acteurs privés, les pouvoirs municipaux et locaux semblent perdre le poids qu'ils avaient su gagner avec les politiques de décentralisation des années 1980-1990 menées pour des raisons internes ou pour appliquer les injonctions internationales au développement durable et son idéal de développement local, que ce soit en Inde (Didelon, Ripert, 2010) ou au Japon (Hein, Pelletier, 2006). Ainsi, les auteurs de ce dossier montrent bien la dépolitisation des débats d'aménagement local dans le cas des smart cities en Inde (Sama Khan, Persis Taraporevala et Marie-Hélène Zérah, 2018, ce dossier), comme à Hong Kong, dans le cas de l'aménagement du nouveau centre d'affaires de Kowloon (Nicolas Douay, 2018b, ce dossier), en Chine continentale (Carine Henriot, 2018, ce dossier), et au Japon en ce qui concerne les smart communities Benoit Granier (2018, ce dossier) et Nicolas Leprêtre (2018, ce dossier). Ce dernier auteur évoque également au sujet des cas japonais la possibilité que les « solutions de l'intelligence » précèdent en réalité la construction des problèmes publics (au sens où l'entend la science politique; Sheppard, 2010). On rejoint donc bien ici l'idée d'auto-réalisation des idéaux (Picon, 2013) et du solutionnisme technologique, qui constitue sans doute le principal facteur de dépolitisation de la fabrique de la smart city en Asie comme ailleurs dans le monde.

Carine Henriot est maître de conférences en aménagement de l'espace et en urbanisme, au sein du département de Génie urbain de Sorbonne universités, Université de technologie de Compiègne, chercheure à I'EA 7284 Avenues, et chercheure associée à l'UMR 8586 Prodig. Ses travaux portent sur la production de la ville chinoise et française, à travers la circulation de modèles urbains, les politiques d'aménagement et les pratiques de la planification territoriale, avec une focalisation sur le numérique et l'énergie. carine.henriot@utc.fr.

Nicolas Douay est Professeur des universités en urbanisme à I'Institut d'Urbanisme et de Géographie Alpine de I'Université Grenoble Alpes et chercheur à l'UMR PACTE. Titulaire d'un doctorat en co-tutelle entre l'Université de Montréal et AixMarseille Université et d'une habilitation à diriger des recherches de Sorbonne Université, il a été maître de conférences à l'Université Paris Diderot de 2009 à 2018, il a aussi séjourné au Centre d'études français sur la Chine contemporaine (CEFC, Hong Kong) grâce à une bourse de post-doctorat Lavoisier en 2008 et 2009, puis de 2015 à 2017 dans le cadre d'une délégation CNRS. Ses recherches se focalisent sur les politiques urbaines, les pratiques de la planification territoriale et les usages $d u$ numérique dans l'urbanisme. nicolas.douay@univ-grenoble-alpes.fr

Benoit Granier est docteur en science politique, chercheur post-doctorant au Centre de Sociologie des Organisations (CSO,

UMR 7116) et chercheur associé à l'Institut d'Asie Orientale (IAO, UMR 5062). Ses travaux portent sur les changements dans

I'action publique et la circulation transnationale des savoirs de gouvernement entre le Japon, les États-Unis et les pays européens. II s'intéresse en particulier aux politiques énergétiques et climatiques et aux programmes de changement des comportements dans les domaines de l'environnement et de la santé publique. 
Il a récemment publié "L'expérimentation sociotechnique fondée sur les sciences comportementales: Un instrument au service de la production de l'acceptabilité sociale? " dans la revue Vertigo (2015), "How are Citizens Involved in Smart Cities? Analysing Citizen Participation in Japanese Smart Communities » dans Information Polity (2016), et "Why do energy conservation policies mobilise the behavioural sciences rather than the social sciences? A few lessons from the emergence of the behaviour change agenda in Japan " dans les Proceedings of the Summer Study of the European Council for an Energy Efficient Economy 2017 (2017). benoit.granier@ens-lyon.fr

Raphaël Languillon-Aussel est docteur et agrégé de géographie, ancien élève de l'École normale supérieure de Lyon et des universités japonaises de Tokyo et de Chuo. Il est actuellement chercheur chargé de cours à l'Université de Genève, et chercheur associé à l'UMR 5600 Environnement, Ville et Société (EVS)

où il conduit des travaux sur quatre thématiques: les politiques de renaissance urbaine et la verticalisation des métropoles; l'impact du vieillissement sur les marchés immobiliers ; les projets d'aménagement olympique; les smart cities.

Co-fondateur du réseau critique Smart, il y coordonne des travaux sur les territoires dits "smart ». II a récemment publié "La stratégie de la smart city au Japon : expérimentations nationales et circulations globales », Echogéo $n^{\circ} 36$ en collaboration avec Nicolas Leprêtre et Benoit Granier. Raphael.Languillon@unige.ch

Nicolas Leprêtre est docteur en science politique de l'École Normale Supérieure de Lyon (Université de Lyon) et chercheur associé à I'Institut d'Asie Orientale (UMR 5062). Il a achevé sa thèse en 2016, sous la direction de la professeure Yveline Lecler, qui porte sur les transformations de l'action publique face à

l'introduction de réseaux électriques intelligents au Japon. Ses recherches incluent l'analyse de l'instrumentation des politiques énergétiques, des relations entre l'État et territoires et du recours à l'expérimentation au sein des politiques publiques. nicolas.lepretre@ens-lyon.fr

\section{BibliograpHIE}

Albino V., Berardi U., Dangelico R. M., 2015, Smart Cities : Definitions, Dimensions, Performance, and Initiatives, Journal of Urban Technology, Vol. 22, № 1, p. 3-21. DOI: 10.1080/10630732.2014.942092

Batty M., 2013, Big Data, Smart Cities and City Planning, Dialogues in Human Geography, n 3-3, p. 274-279.

Bulkeley H., Castán Broto V., 2013, Government by Experiment? Global Cities and the Governing of Climate Change, Transactions of the Institute of British Geographers, Vol. 38, $n^{\circ} 3$, p. 361-375.

Caragliu A., Del Bo C., Nijkamp P., 2009, Smart Cities in Europe, Proceedings of the 3 rd Central European Conference

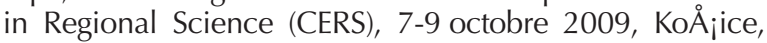
Slovak Republic, p. 49-59. [En ligne] (consulté le 26 octobre 2018) Disponible à l'adresse: http://inta-aivn.org/ images/cc/Urbanism/background\%20documents/01_03_ Nijkamp.pdf

Choay F., 2015, Modèle urbain, in : Choay F., Merlin P. (dir.), Dictionnaire de l'urbanisme et de l'aménagement, Paris: Puf.

Coll J. M., 2014, Beyond Smart Cities: It's Time for Urban Sustainable Development. Notes Internacionals CIDOB, $\mathrm{n}^{\circ}$ 92, June. [On line] (accessed 15 oct. 2018) Available at: https://www.cidob.org/es/publicaciones/serie_de_publicacion/notes_internacionals/n1_92/beyond_smart_cities_ it_s_time_for_urban_sustainable_development

CoutARd O., Rutherford J., 2009, Les réseaux transformés par leurs marges: développement et ambivalence des techniques « décentralisées », Flux, 2009/2-3 (N 76-77), p. 6-13.

DeWIT A., 2014, Japan's Rollout of Smart Cities: What Role for the Citizens?, The Asia-Pacific Journal, vol. 11, Issue 24, Number 2, June 16, 2014. [On line] (accessed 15 oct. 2018) Available at: https://apjjf.org/2014/11/24/AndrewDeWit/4131/article.html
Didelon C., Ripert B., 2010, Un modèle indien du développement des TIC ?, Social and Spatial Dimensions of ICT in India, Netcom journal, Vol. 23, n 3-4, p. 181-200.

Douar N., 2018a, L'urbanisme à l'heure du numérique, Londres : ISTE Édition.

Douar N., 2018b (ce dossier), La « Smart City » comme nouvelle narration des politiques urbaines hongkongaises: le cas du projet urbain de "Kowloon East », Flux, 2018/4 ( No 114), p. 22-37.

Douay N., Henriot C., 2016, La Chine à I'heure des villes intelligentes, L'Information géographique, n² 2016/3 (Vol. 80), p. 89-102. DOI : 10.3917/lig.803.0089

Dupuy G., 2014, L'avenir de la smart city, Urbanisme, $\mathrm{n}^{\circ} 394$, p. 34-35.

Erlinghagen S., Markard J., 2012, Smart grids and the transformation of the electricity sector: ICT firms as potential catalysts for sectoral change, Energy Policy, Vol. 51, December, p. 895-906. DOI:10.1016/j.enpol.2012.09.045

Giffinger R, Fertner C., Kramar H., Kalasek R., Pichler-Milanovic N. et Meijers E., 2007, Smart cities: ranking of European medium-sized cities, Final report for smart-cities.eu, Vienna: Centre of Regional Science (SRF), Vienna University of Technology. [Online] (accessed 15 oct. 2018) Available at: http://www.smart-cities.eu/download/smart_cities_final_ report.pdf

Gram-Hanssen K., 2011, Understanding change and continuity in residential energy consumption, Journal of Consumer Culture, vol.11, p. 61-78. DOI: 10.1177/ 1469540510391725

Granier B., 2018 (ce dossier), Smart cities et gouvernementalisation de la consommation d'énergie domestique au Japon. Le rôle central de l'accident de Fukushima et des pratiques étasuniennes, Flux, 2018/4 (N¹14), p. 56-70.

Granier B., Kudo H., 2016, How are Citizens Involved in Smart Cities? Analysing Citizen Participation in Japanese 
Smart Communities, Information Polity, vol. 21, $\mathrm{n}^{\circ}$ 1, DOI: 10.3233/IP-150367

Greenfield A., 2013, Against the Smart City, 1.3 edition, Kindle Edition, Publish: Do projects.

Hein C., Pelletier P. (eds.), 2006, Cities, autonomy and decentralization in Japan, London, New York: Routledge.

Henriot C., 2018 (ce dossier), La politique chinoise de villes intelligentes : ancrage local d'un modèle urbain globalisé, Flux, 2018/4 (No 114), p. 71-85.

HeRnANDEZ F., 2015, Assembler les projets pour faire territoire. D'une exploration compréhensive des pratiques à la formalisation d'un cadre d'analyse, Mémoire d'habilitation à diriger des recherches en aménagement de l'espace et urbanisme, vol. 2, Université Pierre-Mendès-FranceGrenoble.

HollandS R. G., 2008, Will the real smart city please stand up?, City, Vol. 12, Issue 3, p. 303-320. DOI: 10.1080/13604810802479126

Khan S., Teperevala P., Zérah M.-H., 2018 (ce dossier), Les villes intelligentes indiennes: défis communs et diversification des trajectoires, Flux, 2018/4 (N 114), p. 86-99.

LANGUillon-Aussel R., 2018 (ce dossier), Le programme «smart communities » au Japon. Nouveaux enjeux de pouvoir des ressources et des systèmes d'information urbains, Flux, 2018/4 (No 114), p. 38-55.

Languillon-Aussel R., Leprêtre N., Granier B., 2016, La stratégie de la «smart city » au Japon : expérimentations nationales et circulations globales, EchoGéo, № 36, avril-juin. DOI:10.4000/echogeo. 14598

LEPRÊTRE N., 2018 (ce dossier), Un « modèle national » de ville intelligente? Le rôle de l'État dans la mise en œuvre de réseaux électriques intelligents au Japon, Flux, 2018/4 $\left(\mathrm{N}^{\circ} 114\right)$, p. 9-21.

Meijer A. J., Rodríguez Bolívar M. P., 2016, Governing the Smart City. A Review of the Literature on Smart Urban Governance,
International Review of Administrative Science, Vol. 82, Issue 2, p. 392-408. DOI: 10.1177/0020852314564308

Mellouli S., Luna-Reyes L. F., Zhang J., 2014, Smart government, citizen participation and open data, Information Polity, vol. 19, $n^{\circ} 1-2$, p. 1-4. DOI : 10.3233/IP-140334

Pıcon A., 2013, Smart cities : théorie et critique d'un idéal auto-réalisateur, Paris : Éditions B2.

RIP A., Kemp R., 1998, Technological change, in: Rayner S., Malone E. L. (eds), Human choice and climate change. Vol. II, Resources and technology, Columbus, OH: Battelle Press., p. 327-399.

Rochet C., 2014, Les villes intelligentes, enjeux et stratégies pour de nouveaux marchés, Étude réalisée avec le concours de CERGAM, CESAMES et du Service de coordination à l'intelligence économique (Ministère français des Finances et des comptes publics), Conference Paper, Conférence CMDM, Paris, novembre. DOI: 10.13140/2.1.4598.4962

Sechilariu M., Wang B., Locment F., 2013, Building-integrated microgrid: Advanced local energy management for forthcoming smart power grid communication, Energy and Buildings, vol. 59, p. 236-243.

Sheppard E., 2010, Problème public, in : Boussaguet L., Jacquot S., Ravinet P. (sous la direction de) Dictionnaire des politiques publiques, Paris : Presses de Sciences Po, p. 530-538.

Shove E., 2010, Beyond the ABC: climate change policy and theories of social change, Environment and Planning $A$, vol. 42, n 6, p. 1273-1285. DOI : 10.1068/a42282

Strengers Y., 2013, Smart Energy Technologies in Everyday Life Smart Utopia?, London : Palgrave Macmillan.

Townsend A., 2014, Smart Cities: Big Data, Civic Hackers and the Quest for a New Utopia, New York: W.W. Norton \& Company.

VANOLO A., 2014, Smartmentality: The Smart City as Disciplinary Strategy, Urban Studies, Vol. 51, Issue 5, p. 883-998. DOI : 10.1177/0042098013494427 